\title{
Review Article \\ Energy of the Conduction Band in Near Critical Point Fluids
}

\author{
C. M. Evans ${ }^{1,2}$ and G. L. Findley ${ }^{3}$ \\ ${ }^{1}$ Department of Chemistry and Biochemistry, Queens College-CUNY, Flushing, NY 11367, USA \\ ${ }^{2}$ Department of Chemistry, Graduate Center-CUNY, New York, NY 10016, USA \\ ${ }^{3}$ Department of Chemistry, University of Louisiana at Monroe, Monroe, LA 71209, USA \\ Correspondence should be addressed to C. M. Evans, cherice.evans@qc.cuny.edu
}

Received 6 September 2010; Accepted 6 December 2010

Academic Editor: Anand Pathak

Copyright (C 2010 C. M. Evans and G. L. Findley. This is an open access article distributed under the Creative Commons Attribution License, which permits unrestricted use, distribution, and reproduction in any medium, provided the original work is properly cited.

\begin{abstract}
The study of the evolution of the conduction band in dense gases and supercritical fluids near the critical point has been complicated by a lack of precise experimental measurements. Both photoemission from an electrode immersed in the fluid and field ionization of a molecule doped into the fluid have been used to probe solvent density effects on the energy of an excess electron as a function of fluid number density and temperature. In this perspective, we present recent experimental results that show a strong critical point effect on the minimum conduction band energy near the critical density and temperature of a fluid. We also discuss the recent development of a new theoretical model that advances our understanding of the density and temperature dependence of the conduction band minimum in near critical point fluids.
\end{abstract}

\section{Introduction}

The critical point of a dense gas represents a state of matter under extreme conditions. (In argon, e.g., the critical point occurs at $T_{c}=-122.4^{\circ} \mathrm{C}$ and $p_{c}=48.7$ bar.) In studies of the evolution of the conduction band in insulators-from the low density gas to the liquid to the solid-the unique properties of the critical point have only recently been investigated, as will be described below. Our fundamental understanding of the behavior of electrons in an insulator follows from experimental and theoretical studies on conduction in solid state systems, where translational symmetry allows one to predict accurately the shape and electronic characteristics of both the valence and conduction bands. The energy of an electron at the bottom of the lowest conduction band is the minimum energy $V_{0}$ of the conduction band relative to the vacuum level. In comparison to this picture, a free electron injected into a perturber gas has an energy that can be predicted based on single scattering interactions between the electron and individual perturbers. However, as the density of a perturber gas increases towards fluid densities, the electron/perturber interaction cannot be treated as a single scattering problem. Unlike in a solid, however, there is no long range translational symmetry. Since the density of the fluid can be adjusted dramatically using both pressure and temperature, understanding the minimum energy $V_{0}\left(\rho_{\mathrm{P}}\right)$ of the conduction band of an electron in a fluid of density $\rho_{\mathrm{P}}$ is a complex problem.

\section{Minimum Conduction Band Energy $V_{0}\left(\rho_{\mathrm{P}}\right)$ in Dense Gases}

Two general methods [1-6] are available to measure the minimum energy $V_{0}\left(\rho_{\mathrm{P}}\right)$ of the conduction band in a fluid, namely, photoemission from an electrode placed in the fluid and photoionization of a dopant perturbed by the fluid. Photoemission allows for the direct measurement of $V_{0}\left(\rho_{\mathrm{P}}\right)$ if the photoemission threshold of the metal in a vacuum and in the fluid can be accurately determined. However, contamination of the metal surface and coating of the electrode surface by the fluid make accurate correction of photoemission data problematic (cf. Figure 1(a)). The second method involves the measurement of the photoionization spectrum of a dopant perturbed by fluid. The exact ionization energy of a dopant in a perturbing fluid can be difficult to determine, because the perturbing fluid broadens and shifts the ionization threshold as the density 


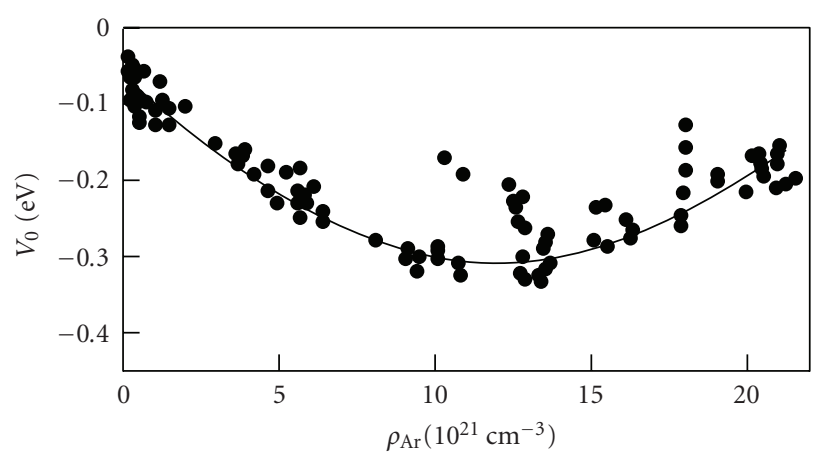

(a)

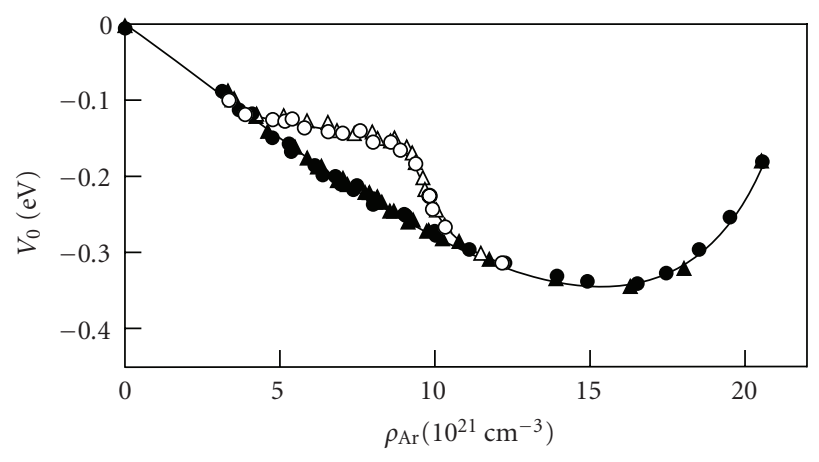

(b)

FIgURE 1: The quasifree electron energy $V_{0}\left(\rho_{\text {Ar }}\right)$ in argon plotted as a function of argon number density $\rho_{\text {Ar }}$. (a) $V_{0}\left(\rho_{\text {Ar }}\right)$ obtained from various photoinjection studies [3-6] at noncritical temperatures. The average error for these measurements is approximately $0.07 \mathrm{eV}$ [3]. (b) $V_{0}\left(\rho_{\text {Ar }}\right)$ obtained from (3) $[2,7-9]$ at noncritical temperatures (solid markers) and on an isotherm near the critical temperature (open markers). The average error for these measurements is approximately $0.02 \mathrm{eV} \mathrm{[7-9].} \mathrm{The} \mathrm{line} \mathrm{in} \mathrm{(a)} \mathrm{is} \mathrm{provided} \mathrm{as} \mathrm{a} \mathrm{guide} \mathrm{to} \mathrm{the} \mathrm{eye.} \mathrm{The} \mathrm{lines}$ in (b) are calculated using the local Wigner-Seitz model (see text for discussion).

increases. This problem can be overcome by measuring the photoionization spectrum of the dopant at two different electric field strengths, since an electric field ionizes high- $n$ Rydberg states converging to the ionization threshold. Thus, taking the difference between two photoionization spectra measured with different applied electric fields (after intensity normalizing the spectra to remove the effects of secondary ionization) yields a peak that represents the Rydberg states ionized by the high electric field $F_{H}$ but not by the low field $F_{L}$. The energy shift $\Delta I$ of the field ionization peak is given by $[1,2,4,7-13]$

$$
\Delta I=-c_{0}\left(F_{L}^{1 / 2}+F_{H}^{1 / 2}\right),
$$

where $c_{0}$ is a dopant-dependent field ionization constant. The zero-field dopant ionization energy $I_{0}\left(\rho_{\mathrm{P}}\right)$ is, therefore, $[1,2,4,7-13]$

$$
I_{0}\left(\rho_{\mathrm{P}}\right)=I_{F}\left(\rho_{\mathrm{P}}\right)+c_{0}\left(F_{L}^{1 / 2}+F_{H}^{1 / 2}\right),
$$

where $I_{F}\left(\rho_{\mathrm{P}}\right)$ is the energy of the maximum of the dopant field ionization peak at the perturber density $\rho_{\mathrm{P}}$. Although dopant field ionization allows one to determine the dopant ionization threshold accurately, this ionization energy is not the minimum energy $V_{0}\left(\rho_{\mathrm{P}}\right)$ of the conduction band of the perturbing fluid. The relationship between the perturbershifted dopant ionization energy $I_{0}\left(\rho_{\mathrm{P}}\right)$ and $V_{0}\left(\rho_{\mathrm{P}}\right)$ is $[1,2$, $4,7-13$ ]

$$
\Delta\left(\rho_{\mathrm{P}}\right)=I_{0}\left(\rho_{\mathrm{P}}\right)-I_{g}=P_{+}\left(\rho_{\mathrm{P}}\right)+V_{0}\left(\rho_{\mathrm{P}}\right),
$$

where $I_{g}$ is the ionization energy of the unperturbed dopant, and $P_{+}\left(\rho_{\mathrm{P}}\right)$ is the ensemble-averaged dopant core/perturber polarization energy, which can be calculated using standard statistical mechanical techniques [1, 2, 7-13]. Figure 1(b) shows an example of $V_{0}\left(\rho_{\mathrm{P}}\right)$ obtained from (3). Clearly, the results from (3) are consonant with those obtained from photoemission measurements. In fact, $V_{0}\left(\rho_{\mathrm{P}}\right)$ determined from the experimental $\Delta\left(\rho_{\mathrm{P}}\right)$ has less scatter, thus making field ionization of high- $n$ dopant Rydberg states an ideal technique for the study of critical point effects (cf. Figures 1 (b) and 2).

\section{Near Critical Point Fluids}

$T_{c}$ is that temperature above which distinct gas and liquid phases do not exist. Above $T_{c}$, the single phase system is generally referred to as a supercritical fluid (SCF) if the pressure is above the critical pressure. In an SCF, the fluid density, viscosity, solubility, and diffusivity can be tailored by adjusting the pressure and temperature. Thus, SCFs constitute controllable, tunable solvents that are currently being employed in the synthesis of various nanoparticles [14-16] having small size distributions with shorter preparation and purification times. The bulk controllability of SCFs has also been used in the pharmaceutical industry $[17,18]$ to create high structural purity organic crystals. The lack of a detailed understanding of the intermolecular interactions in SCFs, however, hampers modeling the more complex reaction dynamics involved in the synthesis of nanomaterials and pharmaceutical compounds in an SCF solvent.

The myriad of factors that affect chemical reactions in near critical point fluids, where the correlation length is essentially unbounded, make the modeling and understanding of chemical processes in these fluids troublesome [19-21]. The difficulty of isolating process control factors in near critical point fluids dictates the study of simple model systems, and therefore we have focused on the energy of the quasifree electron in near critical point fluids. The minimum energy $V_{0}\left(\rho_{\mathrm{P}}\right)$ of the conduction band, obtained from (3), is shown as a function of reduced number density $\rho_{\mathrm{r}} \equiv \rho_{\mathrm{P}} / \rho_{c}$ (with $\rho_{c}$ being the critical number density) for $\mathrm{Ar}$ in Figure 1(b) and for $\mathrm{Kr}, \mathrm{Xe}, \mathrm{CH}_{4}$, and $\mathrm{C}_{2} \mathrm{H}_{6}$ in Figure 2. Clearly, there is little or no temperature dependence in $V_{0}\left(\rho_{\mathrm{P}}\right)$ along noncritical isotherms (solid markers). However, $V_{0}\left(\rho_{\mathrm{P}}\right)$ does vary considerably near the critical temperature and density of the perturber fluid. The variation 


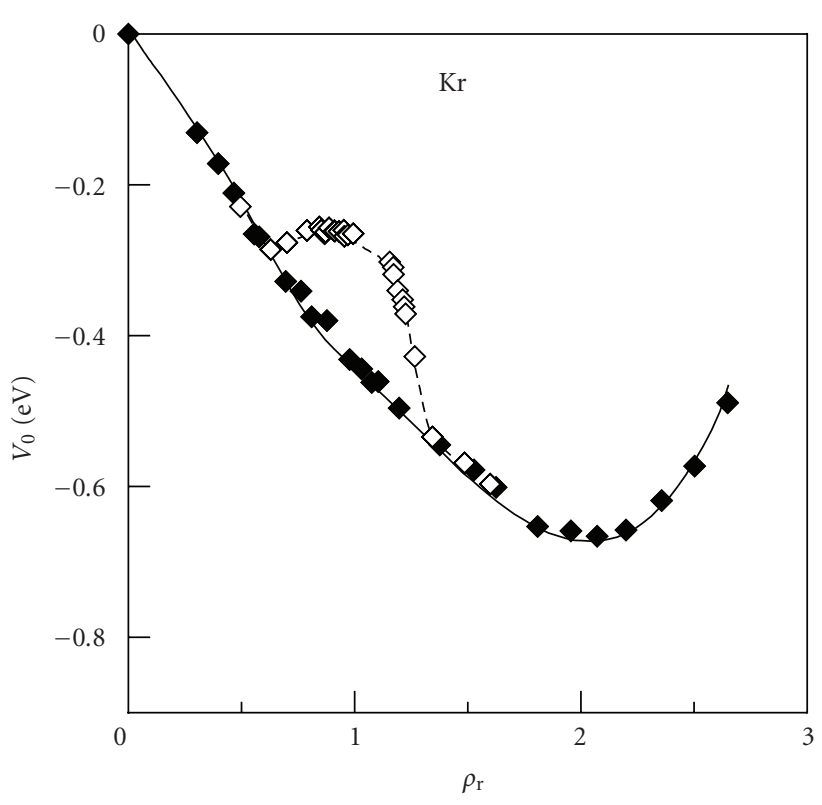

(a)

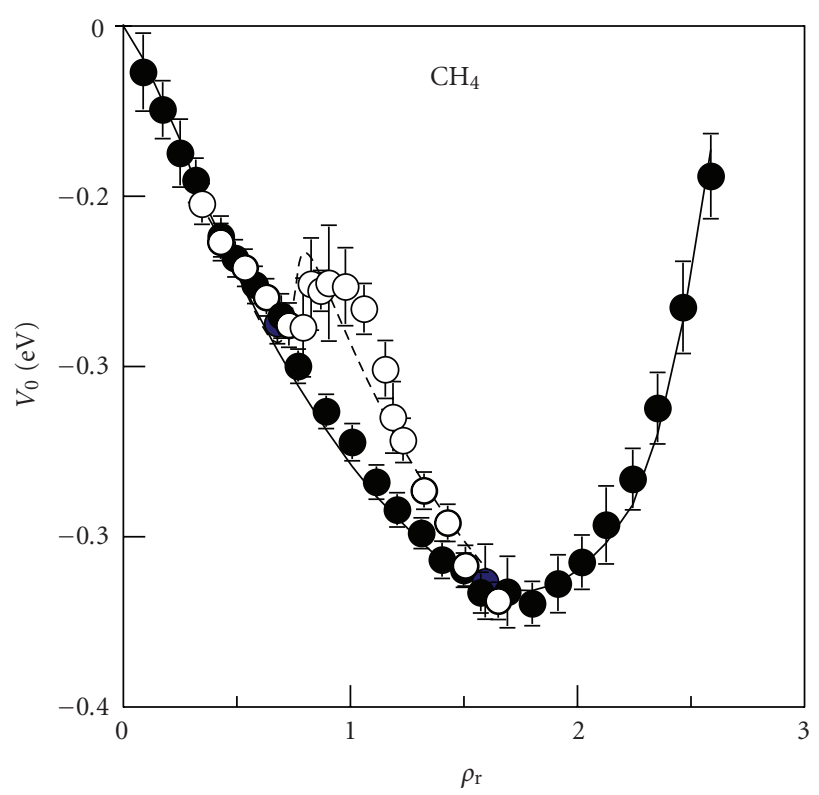

(c)

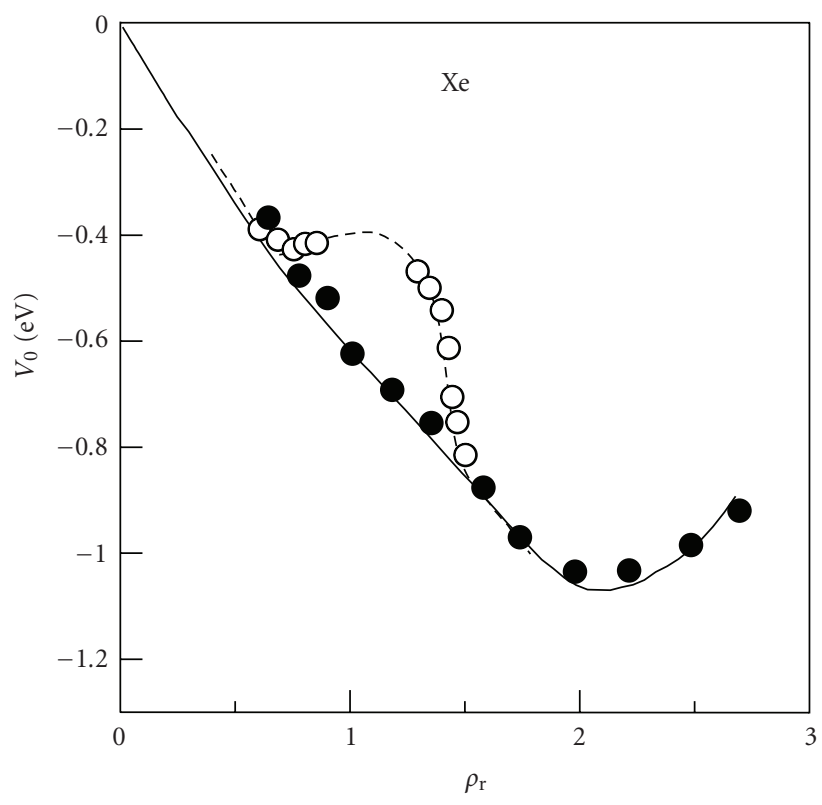

(b)

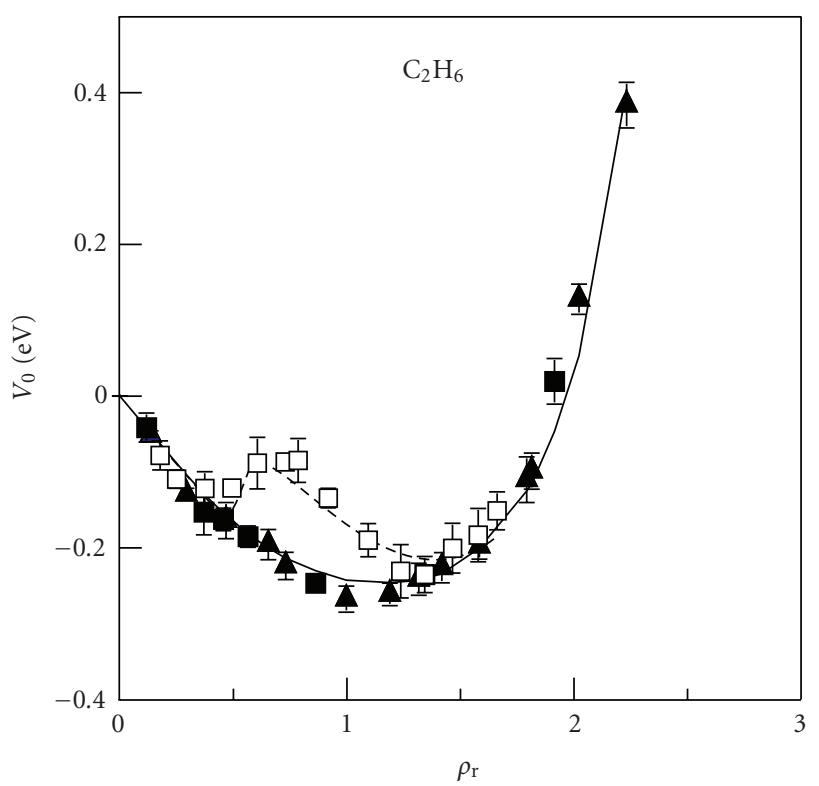

(d)

Figure 2: $V_{0}\left(\rho_{\mathrm{P}}\right)$ plotted as a function of reduced number density $\rho_{\mathrm{r}}$. Minimum energy $V_{0}\left(\rho_{\mathrm{P}}\right)$ of the conduction band, obtained from $(3)$, plotted as a function of reduced number density $\rho_{\mathrm{r}} \equiv \rho_{\mathrm{P}} / \rho_{c}$, where $\rho_{c}$ is the critical density, for $\mathrm{Kr}[2,7,10,12], \mathrm{Xe}^{2}[2,8,12], \mathrm{CH} 4[1,2]$, and $\mathrm{C}_{2} \mathrm{H}_{6}[1,2]$ at noncritical temperatures (solid markers) and on an isotherm near the critical temperature (open markers). The average error in the $\mathrm{Kr}$ and $\mathrm{Xe}$ measurements presented here is approximately $0.02 \mathrm{eV}$ [2]. The error bars for the $\mathrm{CH}_{4}$ and $\mathrm{C}_{2} \mathrm{H}_{6}$ measurements are shown. The lines in these figures are calculated using the local Wigner-Seitz model (see text for discussion).

of $V_{0}\left(\rho_{\mathrm{P}}\right)$ in near critical point SCFs may well have a bearing on the changes in product distribution and reactivity [22] observed in chemical reactions carried out in such systems. Understanding why $V_{0}\left(\rho_{\mathrm{P}}\right)$ changes in a near critical point SCF requires a new model for conduction in a fluid, however.

\section{Local Wigner-Seitz Model [1, 2, 7-13]}

In the local Wigner-Seitz model, which is a recent modification of the original Springett, Jortner, and Cohen model [23], $V_{0}\left(\rho_{\mathrm{P}}\right)$ is given by 


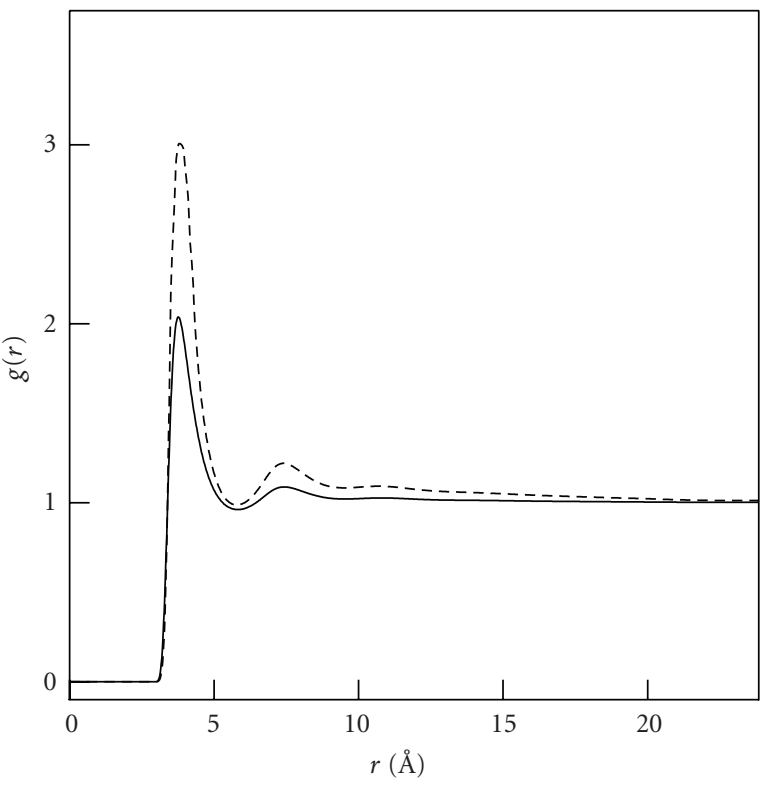

(a)

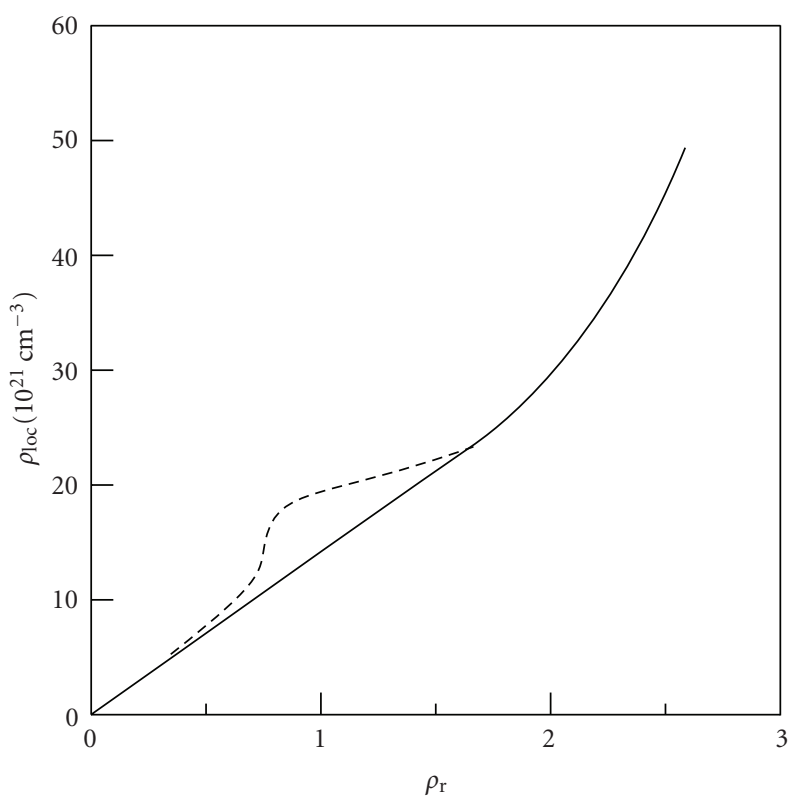

(b)

Figure 3: Critical point effects on the local density. (a) Radial distribution function of argon for the noncritical temperature of $-118^{\circ} \mathrm{C}$ (black) and for the critical temperature of $-122^{\circ} \mathrm{C}$ (red). In both cases, the argon density is the critical density $\left(8.0 \times 10^{21} \mathrm{~cm}^{-3}\right)$. $(\mathrm{b}) \mathrm{The}$ calculated local density $\rho_{\text {loc }}$ plotted as a function of reduced density $\rho_{\mathrm{r}}$ in methane at noncritical temperatures (solid line) and on an isotherm near the critical isotherm (dashed line). Adapted from $[1,2]$.

$$
V_{0}\left(\rho_{\mathrm{P}}\right)=P_{-}\left(\rho_{\mathrm{P}}\right)+E_{k}\left(\rho_{\mathrm{P}}\right)+\frac{3}{2} k_{B} T
$$

where $P_{-}\left(\rho_{\mathrm{P}}\right)$ is the ensemble-averaged electron/perturber polarization energy, $E_{k}\left(\rho_{\mathrm{P}}\right)$ is the zero-point kinetic energy of the quasifree electron, and $(3 / 2) k_{B} T\left(k_{B} \equiv\right.$ Boltzmann's constant) is the thermal energy of the quasifree electron. The average electron/perturber polarization energy is determined using standard statistical mechanical techniques $[2,7]$ :

$$
P_{-}\left(\rho_{\mathrm{P}}\right)=-4 \pi \rho_{\mathrm{P}} \int_{0}^{\infty} g(r) w_{-}(r) r^{2} d r,
$$

where $g(r)$ is the perturber/perturber radial distribution function and $w_{-}(r)$ is the electron/perturber interaction potential. Any critical point behavior in $P_{-}\left(\rho_{\mathrm{P}}\right)$ arises from variations in $g(r)$ near the critical density and temperature.

The zero-point kinetic energy $E_{k}\left(\rho_{\mathrm{P}}\right)$ of the quasifree electron is obtained from solving the Schrödinger equation

$$
\nabla^{2} \psi+\frac{2 m_{e}}{\hbar^{2}}\left[E_{k}-V_{\text {loc }}(r)\right] \psi=0
$$

for the quasifree electron in a dense perturber. In (6), $V_{\text {loc }}(r)$ is a short-ranged potential that accounts for local dynamic polarization of a perturber by the optical electron, $m_{e}$ is the mass of the electron, and $\hbar$ is the reduced Planck constant. The local potential $V_{\text {loc }}(r)$ satisfies an average translational symmetry

$$
V_{\mathrm{loc}}(r)=V_{\mathrm{loc}}\left(r+2 r_{b}\right)
$$

where $r_{b}$ is the interaction range. At any density, the minimum distance between a quasifree electron with low kinetic energy and a single perturber is given by the absolute value of the scattering length $A$. At high perturber number densities, under the assumption that interactions in the first solvent shell dominate the dynamics of the problem, the maximum distance $r_{\ell}$ for a short-ranged interaction is one-half of the spacing between two perturbers in the first solvent shell. This maximum spacing, otherwise known as the local WignerSeitz radius, is

$$
r_{\ell}=\sqrt[3]{\frac{3}{4 \pi g_{m} \rho_{\mathrm{P}}}}
$$

where $g_{m}$ is the maximum of $g(r)$. Thus, the interaction range for the local short-ranged potential is $r_{b}=r_{\ell}-|A|$. By applying these conditions to the asymptotic solutions of (6), the zero-point kinetic energy of the quasifree electron is

$$
E_{k}\left(\rho_{\mathrm{P}}\right)=\frac{\hbar^{2} \eta_{0}^{2}}{2 m_{e}\left(r_{\ell}-|A|\right)^{2}} .
$$

In (9), $\eta_{0}$ is the phase shift induced by the short-ranged potential, and the density dependence arises from $r_{\ell}$. Thus, any critical point behavior in $E_{k}\left(\rho_{\mathrm{P}}\right)$ will result from a change in $r_{\ell}$ near the critical density along the critical isotherm due to density fluctuations, as illustrated in Figure 3(a) for Ar. Similarly, Figure 3(b) presents the maximum local density in the first solvent shell (i.e., $\rho_{\text {loc }}=g_{m} \rho_{\mathrm{P}}$ ) of methane plotted as a function of the reduced perturber number density $\rho_{\mathrm{r}}$. Clearly, the enhancement of the local density at the critical temperature extends to number densities around the critical density, and not just to the critical density itself. 


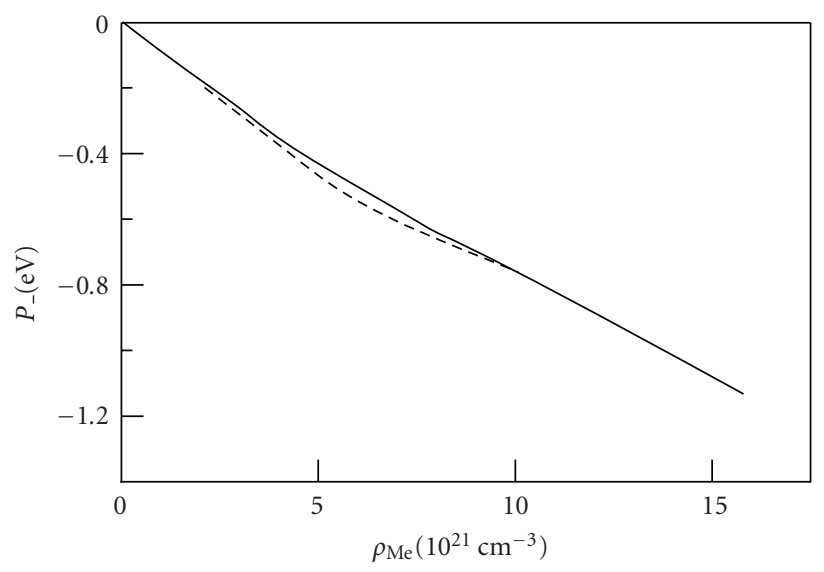

(a)

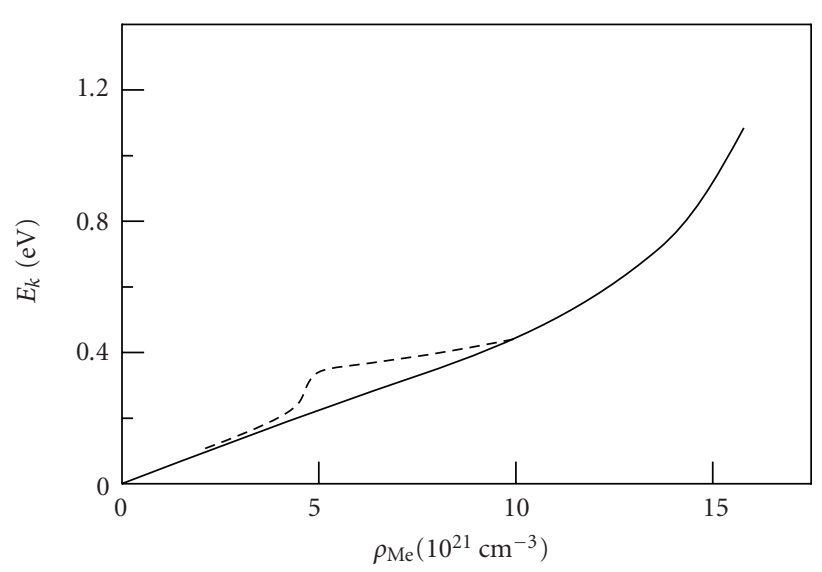

(b)

Figure 4: Local Wigner-Seitz calculation for $\mathrm{CH}_{4}$. The calculated (a) average electron/perturber polarization energy $P_{-}\left(\rho_{\text {Me }}\right)$ and (b) zeropoint kinetic energy $E_{k}\left(\rho_{\mathrm{Me}}\right)$ plotted as a function of the $\mathrm{CH}_{4}$ number density $\rho_{\mathrm{Me}}$, at noncritical temperatures (solid lines), and on an isotherm near the critical temperature (dashed lines). Adapted from $[1,2]$.

(In fact, the critical isotherm turning points that bound the saddle point in the perturber phase diagram coincide with the number densities that delimit the deviations of $\rho_{\text {loc }}$ from the noncritical isotherm values.)

Figure 4 shows the average electron/perturber polarization energy $P_{-}\left(\rho_{\mathrm{P}}\right)$ and the zero-point kinetic energy $E_{k}\left(\rho_{\mathrm{P}}\right)$ for methane at noncritical temperatures and on an isotherm near the critical isotherm of methane. Although $P_{-}\left(\rho_{\mathrm{P}}\right)$ shows a small critical point effect (Figure 4(a)), the critical point variation in $V_{0}\left(\rho_{\mathrm{P}}\right)$ is dominated by the effects on the zero-point kinetic energy $E_{k}\left(\rho_{\mathrm{P}}\right)$ of the quasifree electron (Figure 4(b)), which is dependent on the local density within the first solvent shell through the local Wigner-Seitz radius. Thus, as the local density in the first solvent shell increases, the interaction range for the electron and a single perturber decreases. This decrease in interaction box size increases $E_{k}\left(\rho_{\mathrm{P}}\right)$ near the critical point of the fluid, thus leading to the observed critical point effect.

The lines in Figures 1(b) and 2, which are calculated from the local Wigner-Seitz model described above, accurately fit the experimental data across the entire density range both for noncritical temperatures and on an isotherm near the critical isotherm. In conclusion, the development of dopant field ionization as a technique to extract the minimum energy of the conduction band, $V_{0}\left(\rho_{\mathrm{P}}\right)$, with minimal scatter led to the discovery of a strong critical point effect. This discovery, in turn, has resulted in the development of a new model for electron conduction in dense fluids that represents an order of magnitude improvement over previous theoretical treatments $[1,2,7-13]$.

\section{Acknowledgments}

The University of Wisconsin Synchrotron Radiation Center is funded by NSF DMR-0537588. This work was supported by grants from the Petroleum Research Fund (45728-B6), from the Professional Staff Congress-City University of New York
(60074-34 35), and from the Louisiana Board of Regents Support Fund (LEQSF(2006-09)-RD-A33).

\section{References}

[1] X. Shi, L. Li, G. L. Findley, and C. M. Evans, "Energy of the excess electron in methane and ethane near the critical point," Chemical Physics Letters, vol. 481, no. 4-6, pp. 183-189, 2009.

[2] X. Shi, Energy of the quasi-free electron in atomic and molecular fluids, Ph.D. dissertation, City University of New York, 2010.

[3] R. Reininger, U. Asaf, I. T. Steinberger, and S. Basak, "Relationship between the energy V0 of the quasi-free-electron and its mobility in fluid argon, krypton, and xenon," Physical Review $B$, vol. 28, no. 8, pp. 4426-4432, 1983.

[4] A. K. Al-Omari, Field ionization as a technique to determine electronic properties of fluids, Ph.D. dissertation, University of Wisconsin, Madison, Wis, USA, 1996.

[5] R. A. Holroyd, "Electrons in nonpolar fluids," in Charged Particle and Photon Interactionswith Matter: Chemical, Physicochemical and Biological Consequences with Applications, A. Mozumder and Y. Hatano, Eds., Marcel Dekker, New York, NY, USA, 2004.

[6] I. T. Steinberger, "Band structure parameters of classical rare gas liquids," in Electronic Excitations in Liquefied Rare Gases, W. F. Schmidt and E. Illenberger, Eds., American Scientific Publishers, Valencia, Calif, USA, 2005.

[7] C. M. Evans and G. L. Findley, "Energy of the quasifree electron in argon and krypton," Physical Review A - Atomic, Molecular, and Optical Physics, vol. 72, no. 2, Article ID 022717, pp. 1-12, 2005.

[8] C. M. Evans and G. L. Findley, "Energy of the quasifree electron in supercritical argon near the critical point," Chemical Physics Letters, vol. 410, no. 4-6, pp. 242-246, 2005.

[9] C. M. Evans and G. L. Findley, "Field ionization of $\mathrm{C}_{2} \mathrm{H}_{5} \mathrm{I}$ in supercritical argon near the critical point," Journal of Physics $B$, vol. 38, pp. L269-L275, 2005.

[10] L. Li, C. M. Evans, and G. L. Findley, "Energy of the quasifree electron in supercritical krypton near the critical point," Journal of Physical Chemistry A, vol. 109, no. 47, pp. 1068310688, 2005. 
[11] X. Shi, L. Li, C. M. Evans, and G. L. Findley, "Energy of the quasi-free electron in xenon," Chemical Physics Letters, vol. 432, no. 1-3, pp. 62-67, 2006.

[12] X. Shi, L. Li, C. M. Evans, and G. L. Findley, "Energy of the quasi-free electron in argon, krypton and xenon," Nuclear Instruments and Methods in Physics A, vol. 582, no. 1, pp. 270273, 2007.

[13] X. Shi, L. Li, G. M. Moriarty, C. M. Evans, and G. L. Findley, "Energy of the quasi-free electron in low density Ar and Kr: extension of the local Wigner-Seitz model," Chemical Physics Letters, vol. 454, no. 1-3, pp. 12-16, 2008.

[14] J. P. Cason, K. Khambaswadkar, and C. B. Roberts, "Supercritical fluid and compressed solvent effects on metallic nanoparticle synthesis in reverse micelles," Industrial and Engineering Chemistry Research, vol. 39, no. 12, pp. 4749-4755, 2000.

[15] Y. Zhang and C. Erkey, "Preparation of supported metallic nanoparticles using supercritical fluids: a review," Journal of Supercritical Fluids, vol. 38, no. 2, pp. 252-267, 2006.

[16] C. Burda, X. Chen, R. Narayanan, and M. A. El-Sayed, "Chemistry and properties of nanocrystals of different shapes," Chemical Reviews, vol. 105, no. 4, pp. 1025-1102, 2005.

[17] I. Pasquali, R. Bettini, and F. Giordano, "Supercritical fluid technologies: an innovative approach for manipulating the solid-state of pharmaceuticals," Advanced Drug Delivery Reviews, vol. 60, no. 3, pp. 399-410, 2008.

[18] I. Pasquali, R. Bettini, and F. Giordano, "Solid-state chemistry and particle engineering with supercritical fluids in pharmaceutics," European Journal of Pharmaceutical Sciences, vol. 27, no. 4, pp. 299-310, 2006.

[19] J. F. Brennecke and J. E. Chateauneuf, "Homogeneous organic reactions as mechanistic probes in supercritical fluids," Chemical Reviews, vol. 99, no. 2-3, pp. 433-452, 1999.

[20] S. A. Egorov and E. Rabani, "Chemical equilibrium in supercritical fluids: solvent effects on the dimerization equilibrium constant," Journal of Chemical Physics, vol. 116, no. 19, pp. 8447-8454, 2002.

[21] S. C. Tucker, "Solvent Density Inhomogeneities in Supercritical Fluids," Chemical Reviews, vol. 99, no. 2-3, pp. 391-418, 1999.

[22] O. Kajimoto, "Solvation in supercritical fluids: its effects on energy transfer and chemical reactions," Chemical Reviews, vol. 99, pp. 355-390, 1999.

[23] B. E. Springett, J. Jortner, and M. H. Cohen, "Stability criterion for the localization of an excess electron in a nonpolar fluid," The Journal of Chemical Physics, vol. 48, pp. 2720-2731, 1968, Erratum in The Journal of Chemical Physics, vol. 51, p. 2291, 1969. 

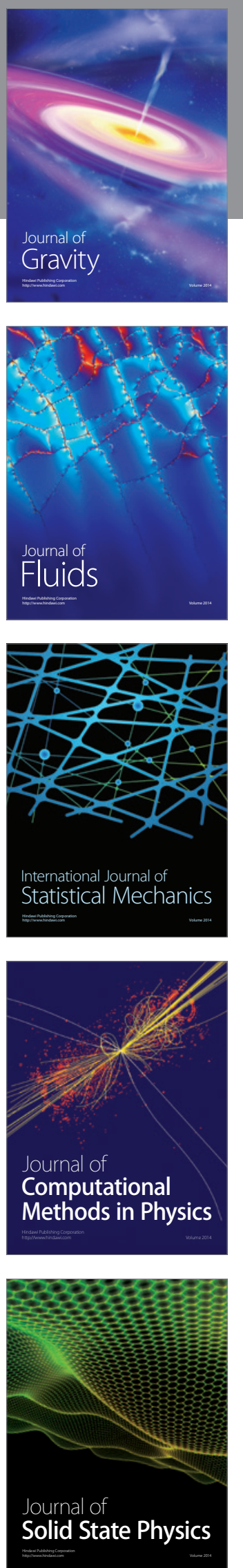

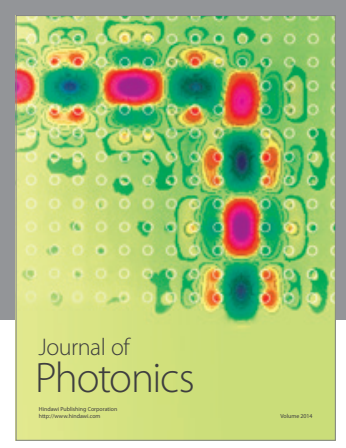

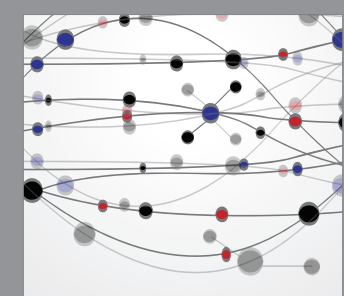

The Scientific World Journal
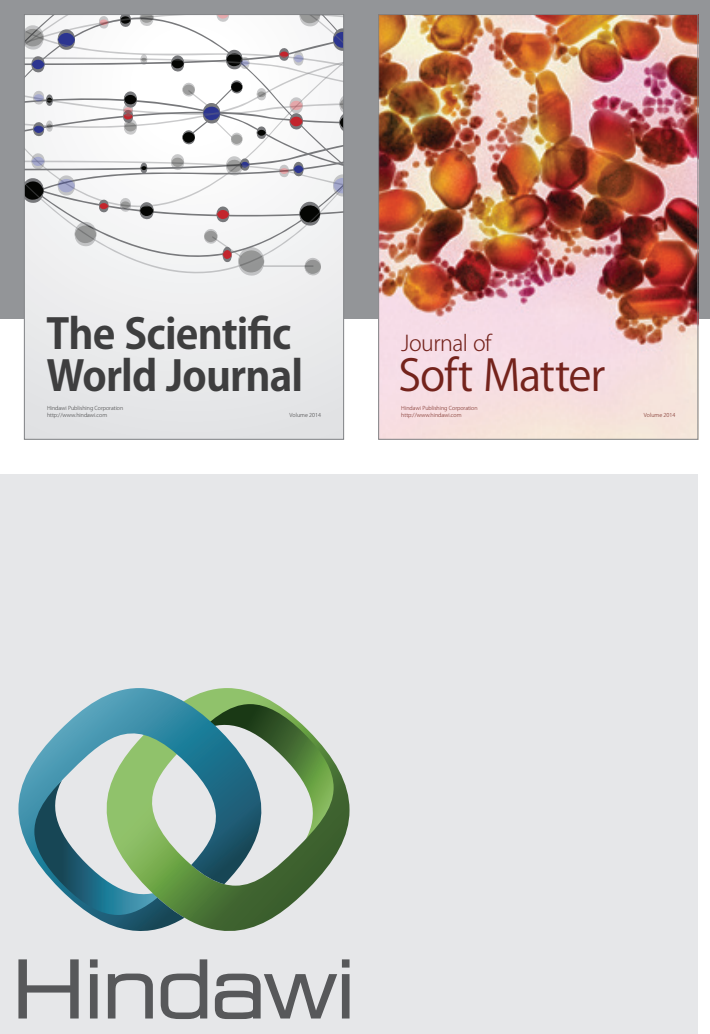

Submit your manuscripts at

http://www.hindawi.com
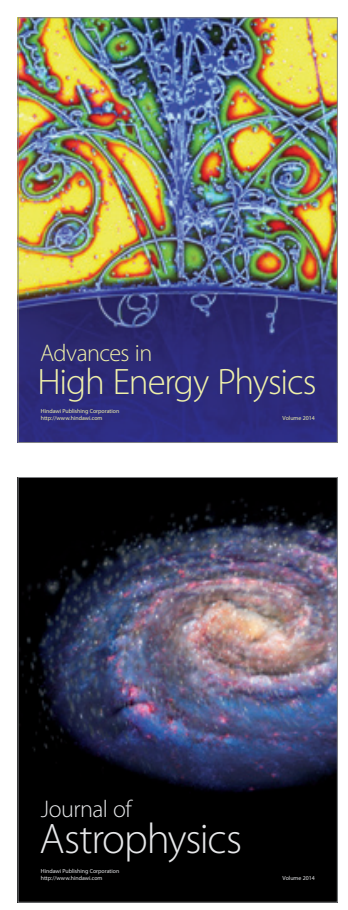
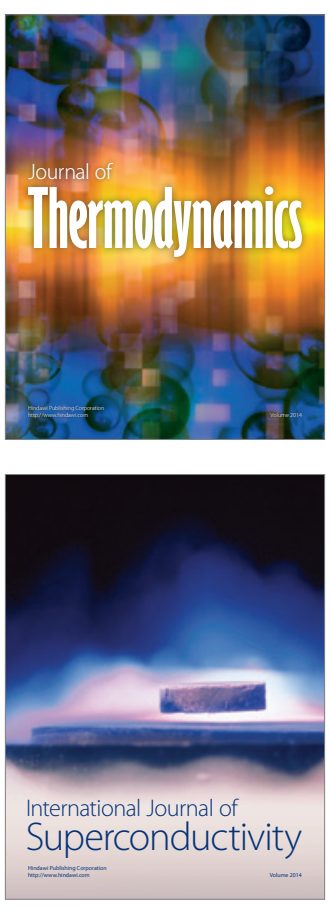
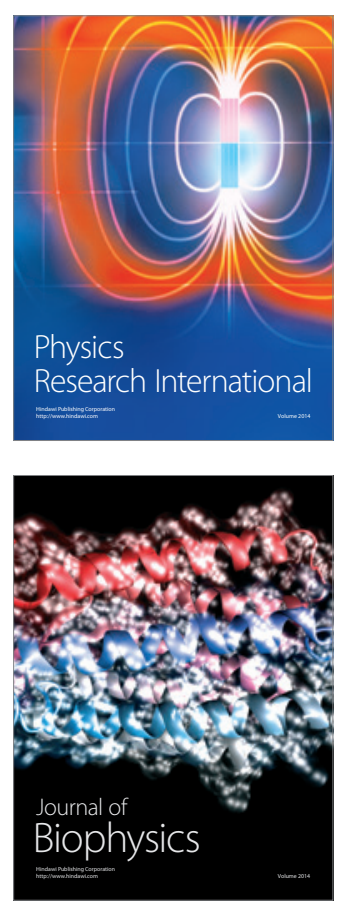
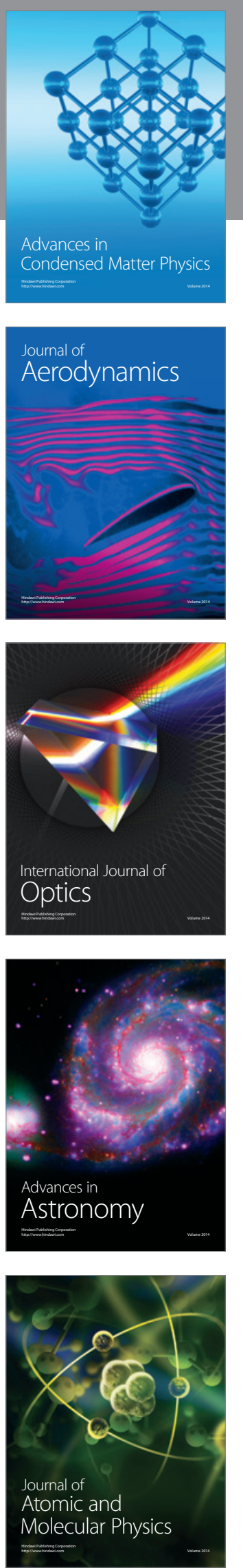